\title{
ALTERACIONES CLÍNICAS Y PARACLÍNICAS EN RECIÉN NACIDOS CON PH ARTERIAL DE CORDÓN UMBILICAL MENOR O IGUAL A 7,18
}

\section{HOSPITAL DE SAN JOSÉ DICIEMBRE 2009 A MARZO 2012}

Maria Claudia Murcia MD*, Sergio Velandia MD**, Estrella Duran MD***, Maria Carolina Uribe MD***, Dolly García MD***, Nathalia Saavedra MD****, María Alejandra Suárez MD****

\section{Resumen}

La depresión neonatal moderada y la asfixia perinatal son poco frecuentes en nuestro medio (2\% en la unidad neonatal). El pH arterial del cordón umbilical $\leq 7.00$ implica mayor riesgo de compromiso multiorgánico, aunque no es claro el pronóstico entre 7.00 y 7.18. El objetivo es describir la frecuencia de alteraciones clínicas y paraclínicas en los neonatos con Apgar $\leq 6$ al primer minuto, cuyos gases arteriales de cordón cursaron con $\mathrm{pH} \leq 7.18$ en el período diciembre 2009 a marzo 2012 en el Hospital San José de Bogotá DC. Metodología: estudio descriptivo prospectivo relativo a la frecuencia de alteraciones por sistemas en los neonatos que cumplieron criterios de inclusión. También se registraron los fallecimientos. Resultados: se incluyeron 52 pacientes, 45 con pH entre 7,11 y 7,18. La alteración más frecuente fue hepática, la dishidrogenia láctica estuvo elevada (100\%) en quienes se realizó la medición, dos casos desarrollaron ecefalopatía (pH 6,96 y 7,13), y no se registró enterocolitis necrosante en dos hubo compromiso renal (oliguria). Cinco fallecieron durante el estudio con $\mathbf{p H} \leq \mathbf{7 . 0 0}$. Conclusiones: aaunque el pH $\leq 7.00$ se considera la mejor evidencia de asfixia perinatal y es predictor de morbimortalidad, los pacientes entre pH 7.00 y 7.18 requieren evaluación y vigilancia clínica porque presentan alteraciones multisistémicas de gravedad variable e incluso muerte.

Palabras clave: gases arteriales de cordón umbilical, pH, recién nacido, depresión neonatal, asfixia perinatal, complicaciones multisistémicas

Abreviaturas: $\mathbf{R N}$, recién nacido; DN; depresión neonatal; AP, asfixia perinatal.

\section{CLINICAL AND LABORATORY ALTERATIONS IN NEWBORNS WITH A CORD ARTERIAL PH LESS OR EQUAL TO 7.18 \\ HOSPITAL DE SAN JOSÉ DECEMBER 2009 TO MARCH 2012}

\section{Abstract}

Moderate neonatal respiratory depression and perinatal asphyxia are uncommon in our setting (2\% at the neonatal unit). Arterial cord $\mathbf{p H}<\mathbf{7 . 0 0}$ infers a higher risk of multisystem complications, although prognosis is still not clear for $\mathrm{pH}$ values between $\mathbf{7 . 0 0}$ and 7.18. Objective: to describe clinical and laboratory alterations in neonates with a score $<6$ on the one-minute Apgar assessment and cord arterial pH $<7.18$, between December 2009 and March 2012 ,

Fecha recibido: junio 15 de 2012 - Fecha aceptado: agosto 28 de 2012

* Pediatra neonatóloga, Jefe del Depto. de Pediatría Hospital de San José, 2008-201I. Bogotá DC, Colombia.

** Pediatra Adscrito, Hospital de San José. Bogotá DC, Colombia.
*** Pediatra, Hospital de San José. Bogotá DC, Colombia.

**** Residente III de Pediatría. Fundación Universitaria de Ciencias de la Salud. Bogotá DC, Colombia. 
at Hospital San José de Bogotá DC. Methodology: a descriptive prospective study on the frequency of multisystem involvement in neonates who met the inclusion criteria. Deaths were also recorded. Results: 52 patients were included, 45 had a pH between 7.11 and 7.18. Liver disorders and elevated lactate dehydrogenase (100\%), when measured, were the most common manifestations. Two cases developed encephalopathy (pH 6.96 and 7.13), two presented renal failure (oliguria). Necrotizing enterocolitis was not evidenced. Five babies with a cord $\mathrm{pH}<7.00$ died during the trial. Conclusions: although a cord $\mathrm{pH}<7.00$ is considered the best evidence of perinatal asphyxia and constitutes a morbidity and mortality predictor, patients with a $\mathrm{pH}$ value between 7.00 and 7.18 require evaluation and monitoring, for they present with multisystem involvement of varied severity including death.

Rey words: umbilical cord arterial gases, pH, newborn, neonatal depression, perinatal asphyxia, multisystem complications.

\section{Introducción}

La asfixia es definida como una alteración del intercambio de gases que lleva a cambios bioquímicos como hipoxemia, hipercapnia y acidosis metabólica. El feto que padece un episodio tiene el riesgo de desarrollar encefalopatía hipóxica isquémica y compromiso multiorgánico. ${ }^{1}$ Sigue siendo una importante causa de mortalidad y morbilidad a largo plazo y su incidencia varía entre 1 a $5 \%$ en los países en desarrollo., Se relaciona en especial con la edad gestacional y el peso al nacer. En neonatos menores de 36 semanas de gestación la incidencia es de $9 \%$ y en los a término se estima en $0.5 \%$. Aumentada en hijos de madres diabéticas o toxémicas, en restricción del crecimiento intrauterino y en presentación de pelvis, causa el $20 \%$ de la mortalidad perinatal.

La incidencia de la encefalopatía hipóxico isquémica principal alteración de la AP ocurre entre dos y nueve por cada mil nacidos vivos. Se considera que hasta el $17 \%$ de las parálisis de los lactantes a término se asocian con eventos hipóxicoisquémicos neonatales. Las secuelas de la AP sin presencia de parálisis cerebral se presentan hasta en el $42 \%$ de los casos. El daño por hipoxia e isquemia afecta cualquier tejido, existen reportes en la literatura de compromiso renal en el $50 \%$ de los pacientes, seguido de sistema nervioso central (28\%), cardiovascular, $(25 \%)$ y pulmonar $(23 \%){ }^{4}$

El cuadro clínico depende del compromiso multisistémico, puede cursar con alteraciones cardiovasculares (hipotensión, taquicardia, pobre perfusión, necrosis miocárdica, insuficiencia cardíaca congestiva) ${ }^{5}$, renales (necrosis tubular y/o insuficiencia renal aguda, oliguria transitoria en las primeras 24 horas, oliguria persistente 36 horas o más) ${ }^{4}$, gastrointestinales (enterocolitis que puede ocurrir hasta los cinco o siete días de edad) ${ }^{6}$, pulmonares (hipertensión pulmonar persistente, aspiración de meconio) ${ }^{7}$ y hematológicas (trombocitopenia). ${ }^{2,8}$

Los criterios diagnósticos de encefalopatía del RN por evento hipóxico incluyen acidemia mixta o metabólica severa, demostrada en gases arteriales de cordón umbilical con $\mathrm{pH}<7.00$, Apgar $\leq 5$ más de cinco minutos, manifestaciones neurológicas en el período neonatal inmediato (convulsiones, hipotonía, coma) y/o evidencia de disfunción multiorgánica. ${ }^{2,7,9,10}$ En nuestra unidad de recién nacidos la DN moderada se define cuando existe Apgar $\leq 6$ al minuto y los cinco minutos, $\mathrm{pH}$ de gases arteriales de cordón umbilical menor $<7.18$ y $>7,0$, y base exceso de -15 a 19 con el paciente asintomático a los diez minutos.

El protocolo varía dependiendo si se trata de DN moderada o AP. En el primer caso se hospitaliza el paciente, se suspende la vía enteral durante 24 horas dado el riesgo de enterocolitis ${ }^{6}$ y se realizan estudios de extensión para descartar compromiso multisistémico, así como monitoreo neurológico estricto. En AP se procede igual pero se suspende la alimentación enteral durante 72 horas. El RN con $\mathrm{pH}$ a $\leq 7.18$ presenta mayor riesgo de compromiso neurológico y multiorgánico en comparación con aquellos con valores superiores, 
siendo esto importante en el grupo con $\mathrm{pH} \leq 7.00$ (que clasificamos como asfixia perinatal) y el de mayor incidencia de alteraciones. ${ }^{1.9}$ Sin embargo de los cinco pacientes que fallecieron durante el estudio solo uno presentó $\mathrm{pH}<7.00$.

El objetivo del estudio es determinar la frecuencia de alteraciones en los casos de DN moderada y aquellos con AP para establecer la ocurrencia de estas en el grupo con $\mathrm{pH}$ entre 7.01 y 7.18 , evaluando la pertinencia del manejo actual en nuestra unidad para evitar el sobretratamiento, así como establecer la mortalidad ante $\mathrm{pH} \leq 7.18$.

\section{Métodos}

Estudio descriptivo prospectivo al que ingresaron los RN del Hospital de San José con DN moderada o AP del $1^{\circ}$. de diciembre de 2009 al 31 de marzo de 2012, usando como criterios de inclusión: RN con Apgar $\leq 6$ al minuto (de acuerdo con la frecuencia cardíaca, tono, irritabilidad refleja, color, respiración), gases arteriales de cordón umbilical con $\mathrm{pH} \leq 7.18$ (muestra obtenida por el pediatra o neonatológo encargado de adaptación neonatal de acuerdo con los criterios del Colegio Americano de Obstetras y Ginecólogos ${ }^{3}$, con doble cierre, extracción con jeringa con heparina, obtención de sangre de arteria y vena umbilicales para evitar sesgo de medición por el origen de la muestra, procesada en un lapso menor de 60 minutos), $\mathrm{RN}$ con peso $\geq 1.500$ gramos y edad gestacional $\geq 34$ semanas por Ballard. Los criterios de exclusión fueron sospecha o evidencia de una malformación mayor (consecuencias estéticas importantes o que requiera atención médica obligada incluyendo tratamiento quirúrgico) por diagnóstico clínico o paraclínico, madre con trombocitopenia $\leq 100.000$, o bien sospecha o confirmación de infección por toxoplasmosis, rubéola, citomegalovirus, herpes, sífilis o VIH que al nacer el RN presente signos de infección congénita con afección de uno o más órganos (calcificaciones cerebrales, microcefalia, hepatomegalia). Aquellos que fueron incluidos se hospitalizaron para estudio paraclínico y seguimiento clínico.

A las 24 horas de vida se realizaron los siguientes paraclínicos: hemograma con recuento automatiza- do de plaquetas, nitrógeno ureico (BUN), creatinina sérica, aspartato aminotransferasa (TGO), alanino aminotransferasa (TGP), calcio, bilirrubina directa, deshidrogenasa láctica (LDH) y monitoreo hemodinámico y neurológico. De acuerdo con las alteraciones halladas se definieron las siguientes variables dentro del estudio: $\mathrm{pH}$, medido en gases arteriales de cordón con dos decimales teniendo en cuenta valores $\leq 7.18$; género; puntuación Apgar realizada por el pediatra y/o neonatólogo en sala de partos al primer y quinto minutos de vida; peso; vía del parto; presentación; alteración neurológica, cualquiera según la clasificación de Sarnat (dos y tres) aplicada a las 24, 48 y 72 horas de vida ${ }^{11}$; alteración hepática, definida como la elevación de enzimas (TGO/TGP) con valor $\geq 100$ UI/l y/o LDH más de 1,5 veces el valor del límite superior del normal y/o elevación de la bilirrubina directa $>0,20 \%$ del total ${ }^{12,13}$; alteración muscular, incluye valor de LDH elevado; alteración miocárdica, se presenta cuando existe elevación de la troponina $T$ $>0,2 \mathrm{Ug} / \mathrm{l}$ y/o alteración en el ecocardiograma (hiper tensión pulmonar, insuficiencia tricúspidea o mitral, o bien lesión miocárdica hipóxico isquémica) ${ }^{5,14-16}$; alteración renal, cuando la creatinina es $\geq 1,5 \mathrm{mg} / \mathrm{dl}$ a las 24 horas de nacido y/o un gasto urinario inferior a $1 \mathrm{ml} / \mathrm{k} /$ hora. ${ }^{17}$ Trombocitopenia, si el recuento manual de plaquetas es $<100.000 / \mathrm{dl}^{8}$; enterocolitis necrosante, si las manifestaciones clínicas son compatibles con la clasificación de Bell modificada ${ }^{18}$; alteración respiratoria, cuando requiere $\mathrm{FIO}_{2} 0.40$ por más de cuatro horas y/o requerimiento de intubación orotraqueal en cualquier momento desde el nacimiento y durante el tiempo de seguimiento; hipoglicemia, definida como la presencia de glucometría menor a $47 \mathrm{mg} / \mathrm{dl}$, cada ocho horas, durante los tres primeros días de vida ${ }^{19}$; hipocalcemia, cuando el valor de calcio total es $<7$ $\mathrm{mg} / \mathrm{dl}^{20}$ y mortalidad, si el paciente fallece durante el seguimiento hospitalario.

Se realizó un análisis descriptivo evaluando las diferentes variables establecidas que son relacionadas con el compromiso multisistémico y el pH analizado en gases arteriales del cordón al momento del nacimiento. En el análisis de la información se usó el software estadístico Stata 10 y Spad $7.0 \mathrm{El}$ protocolo tuvo la aprobación del comité de investigaciones y ética de la 
Facultad de Medicina de la Fundación Universitaria de Ciencias de la Salud y la investigación fue clasificada sin riesgo.

Para los reclutados entre diciembre 2009 y abril 2010 no se cuenta con la totalidad de los datos para cada variable, por lo tanto se consideraron como pacientes piloto. La información tabulada corresponde a los datos de 52 pacientes incluidos en el proyecto del período mayo 2010 a marzo 2011 (Figura 1).

Las características poblacionales como la igual proporción en la vía del parto, presentación y género se describen en la Tabla 1. La mayoría de manifestaciones clínicas y paraclínicas se presentan en la población de pacientes con DN moderada (Tabla 1).

La elevación de lactato deshidrogenasa fue la alteración más frecuente: $100 \%$ de los pacientes en quienes se realizó la medición. las enzimas hepáticas TGO/ TGP, estuvieron elevadas en el $28.8 \%$ de la población con DN y $28.5 \%$ con AP, sin manifestaciones clínicas de falla hepática. El $15.3 \%$ presentó trombocitopenia leve sin manifestaciones de sangrado activo; en cuanto a las manifestaciones renales el 3,8\% presentaron compromiso durante la estancia hospitalaria (Tabla 2).

Las alteraciones renales registradas fueron oliguria en dos casos, sin valores de creatinina superiores a 1.4 mg\% a las 24 horas. No se observaron casos de enterocolitis necrosante. En cinco se encontró elevación de troponina I, de ellos uno con cambios en el ecocardiograma y otro con $\mathrm{pH}<7.01$. Tres tenían anormalidad en el ecocardiograma sin elevación de troponina.

Las manifestaciones neurológicas se presentaron en dos pacientes de los cuales uno murió. Se registraron cinco fallecimientos que se caracterizan en la Tabla 3 , en la que se puede observar que solo uno presenta $\mathrm{pH}<7$, sin embargo es el que presenta mayor compromiso clínico. El otro con alteración neurológica y que no muere, presenta mayor compromiso clínico comparado con los demás.

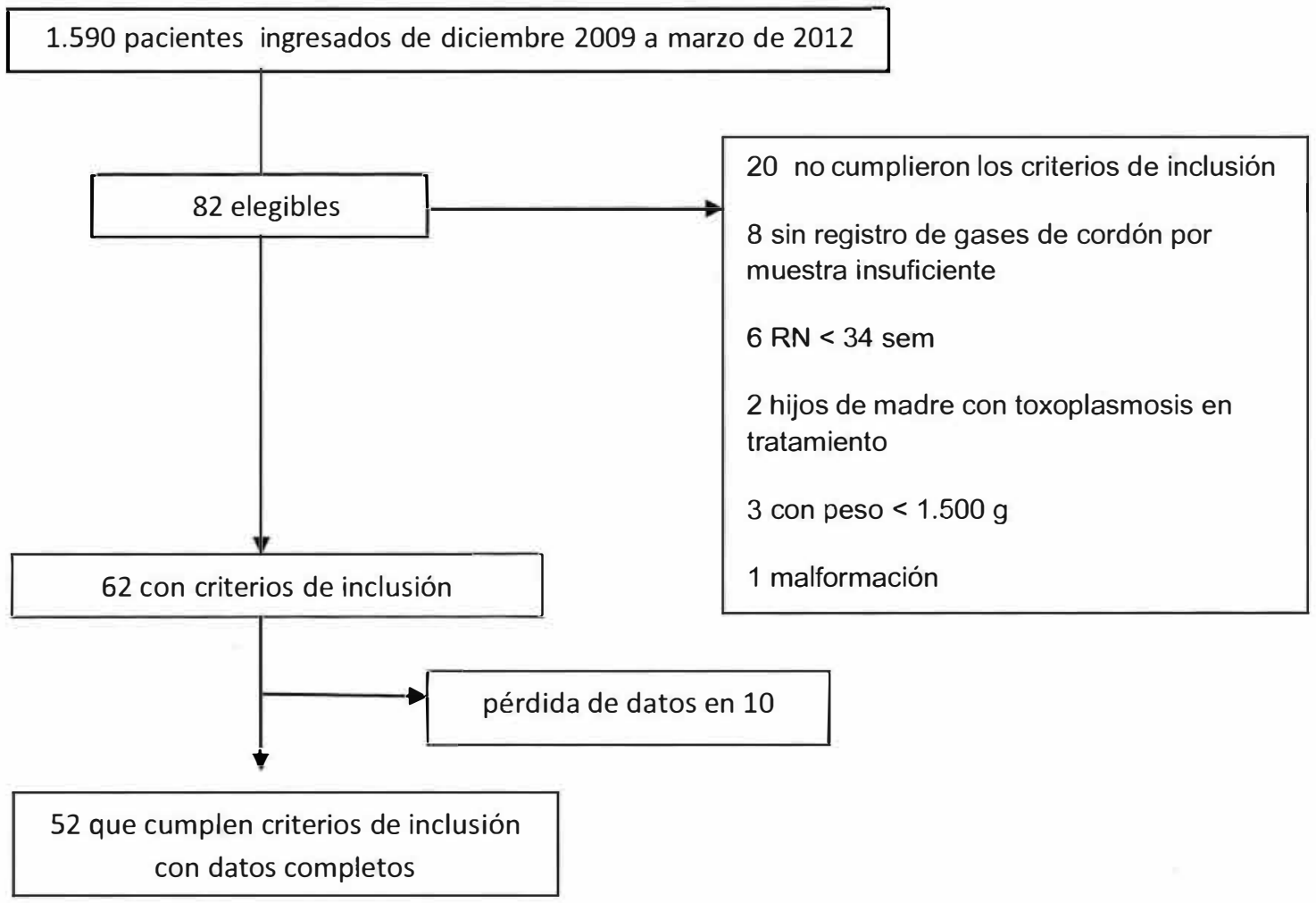

Figura I. Mecanismo de selección. 


\begin{tabular}{|c|c|c|}
\hline \multicolumn{3}{|c|}{ Tabla I. Características de la población } \\
\hline Variable & N & $\%$ \\
\hline Masculino & 36 & $(69.2)$ \\
\hline Presentación & & \\
\hline cefálica & 47 & $(90.3)$ \\
\hline de pelvis & 3 & $(5.7)$ \\
\hline otra & 2 & $(3.8)$ \\
\hline Vía & & \\
\hline vaginal & 26 & $(50.0)$ \\
\hline cesárea & 26 & $(50.0)$ \\
\hline Variable & Promedio & DS \\
\hline Edad Gestacional & 38.4 & $(1.3)$ \\
\hline min/max & 34 & 41 \\
\hline Peso en gramos & 2978.1 & $(443.0)$ \\
\hline min/max & 1760 & 3775 \\
\hline pH & 7.06 & $(0.07)$ \\
\hline min/max & 6.91 & 7.18 \\
\hline Días de hospitalización & 6 & $(3.0)$ \\
\hline min/max & 3 & 17 \\
\hline
\end{tabular}

En cuanto al comportamiento del $\mathrm{pH}$ el valor mínimo encontrado es 6.90 y el máximo corresponde al punto de corte de 7.18; la mayoría de alteraciones se presentan con pH entre 7.11 y 7.18, como son alteración pulmonar (requerimiento de Fio2 $>40 \%$ ), hepática (elevación de TGO/TGP/LDH) y cardíaca (troponina superior a $0.02 \mathrm{ng} / \mathrm{dl}$ ), siendo menos frecuentes las neurológicas, hematológicas y metabólicas.

\section{Discusión}

En pacientes con DN moderada y AP el compromiso multisistémico es variable, y resulta del fenómeno hipóxico isquémico. Es interesante observar el comportamiento de nuestros pacientes, ya que la mayoría de las alteraciones se presentaron con $\mathrm{pH}$ entre 7.11 y 7.18 , considerado por nosotros como DN moderada sin asfixia y corresponden a manifestaciones renales, pulmonares y hepáticas. El compromiso de al menos un órgano se encuentra en todos los RN (hepático/muscular representado por $\mathrm{LDH}$ ), dos casos con cambios

\begin{tabular}{|c|c|c|}
\hline Alteración & Frecuencia & (\%) \\
\hline Neurológica & 2 & (3.8) \\
\hline \multicolumn{3}{|l|}{ Miocárdica } \\
\hline $\begin{array}{l}\text { ecocardiograma alterado } \\
\text { (HTP* ó disfuncion ventricular) }+\end{array}$ & 2 & $(4.3)$ \\
\hline troponina & 5 & (9.6) \\
\hline Promedio/min-max (ng/ml) & 0.095 & $0-0.88$ \\
\hline $\begin{array}{l}\text { Ecocordiagrama alterado }+ \\
\text { troponina elevada }\end{array}$ & 1 & $(2.1)$ \\
\hline Respiratoria & 15 & $(28.8)$ \\
\hline \multicolumn{3}{|l|}{ Hepática } \\
\hline LDH§ & 50 & $(100.0)$ \\
\hline promedio/min-max (U/I) & 3663 & $972-30162$ \\
\hline TGO & 11 & $(21.1)$ \\
\hline promedio/min-max (U/ml) & 89,7 & $33-380$ \\
\hline TGP & 2 & (3.8) \\
\hline promedio/min-max (U/ml) & 37,3 & $12-257$ \\
\hline Renal & 2 & (3.8) \\
\hline Enterocolitis & 0 & 0 \\
\hline Hipocalcemia** & 4 & (7.6) \\
\hline promedio/min-max (mEq/l) & 8.23 & $5.9-10.5$ \\
\hline Hipoglicemia & 2 & (3.8) \\
\hline Trombocitopenia & 8 & $(15.3)$ \\
\hline promedio/min-max & 216627 & $\begin{array}{l}107000- \\
364000\end{array}$ \\
\hline
\end{tabular}

*HTP, hipertensión pulmonar; † datos de 46 pacientes; $\S$ datos de 50 pacientes; **datos de 50 pacientes

en al menos ocho variables con $\mathrm{pH}$ de 7.13 y 6.90 De los cinco fallecimientos uno cursó con $\mathrm{pH}<7.00$.

Dos pacientes presentaron este compromiso con es. tadio II según la escala de clasificación de Sarnat uno con episodio convulsivo en las primeras 24 horas persistencia de hipotonía por 72 horas y recuperaciór posterior, con pH en sangre arterial de cordón umbili cal de 7.13, y el segundo con $\mathrm{pH}$ de 6.96. Al compara los resultados con reportes de la literatura ${ }^{18}$ la altera ción del sistema nervioso central está presente en 72\% de los niños, de estos $41 \%$ con estadio Sarnat I, 21\% estadio II y $10 \%$ estadio III, estando las convulsione presentes en $19 \%$ de los neonatos.

Los signos de daño miocárdico o disfunción cardio vascular se produjeron en 50 a $80 \%$ de los niños col 


\begin{tabular}{|c|c|c|c|c|c|c|c|c|c|}
\hline \multirow{2}{*}{ Paciente } & \multirow{2}{*}{ pH } & \multirow{2}{*}{ Muerte } & \multicolumn{5}{|c|}{ Alteración } & \multirow{2}{*}{ renal } & \multirow{2}{*}{ ECN } \\
\hline & & & miocárdica & respiratoria & neurológica & hepática & hematológica & & \\
\hline 1 & 7 & $\mathrm{Si}$ & No & Si & No & $\mathrm{Si}$ & $\mathrm{Si}$ & No & No \\
\hline 2 & 7.18 & Si & No & $\mathrm{Si}$ & No & No & No & No & No \\
\hline 3 & 7 & $\mathrm{Si}$ & No & $\mathrm{Si}$ & No & No & No & No & No \\
\hline 4 & 7.16 & $\mathrm{Si}$ & No & No & No & $\mathrm{Si}$ & $\mathrm{Si}$ & No & No \\
\hline 5 & 6.96 & $\mathrm{Si}$ & No & $\mathrm{Si}$ & $\mathrm{Si}$ & $\mathrm{Si}$ & No & $\mathrm{Si}$ & No \\
\hline 6 & 7.13 & No & $\mathrm{Si}$ & $\mathrm{Si}$ & $\mathrm{Si}$ & $\mathrm{Si}$ & No & No & No \\
\hline
\end{tabular}

AN. ${ }^{5}$ En este estudio, ocho presentan compromiso cardíaco dado por elevación de troponina o alteración ecocardiográfica (hipoquinesia difusa de la pared libre del ventrículo e hipertensión pulmonar).

La troponina es un predictor útil de isquemia miocárdica y solo uno presentó alteración simultánea de troponina y ecocardiograma, con $\mathrm{pH}$ de 7.13 , otro con troponina elevada tenía $\mathrm{pH}<7.00$ (6.90), los demás con criterios de DN moderada, sin evidencia de choque durante el seguimiento. En nuestro estudio el $9.6 \%$ de los pacientes presentaron alteración miocárdica ya sea por troponina o ecocardiograma; comparado con el estudio de Shah, Riphagen y colaboradores $^{21}$ en donde el $62 \%$ la presentó y los reportes de Shankaran de $50 \%$ y $78 \%$ en el de Hankins y colaboradores. ${ }^{22}$

El paciente asfixiado tiene mayor riesgo de desarrollar hipertensión pulmonar persistente, cursa con hipoxia y acidosis, manifestándose por aumento de la resistencia vascular pulmonar. Se encontró que el $28.8 \%$ requiere Fio2 $>0.4$ por más de cuatro horas, $8.6 \%$ presentan hipertensión pulmonar y $9.6 \%$ requieren ventilación mecánica invasiva; esta alteración está presente en el grupo de DN. Difiere de los reportes de la literatura de Shah y colaboradores y Shankaran con incidencias de compromiso pulmonar hasta de $86 \%$ en AP. ${ }^{21}$ Por el contrario, Ancel, Garcia y colaboradores ${ }^{18}$ estudiaron 85 pacientes con similares características a nuestra población, observando compromiso en $26 \%$ de los cuales $7 \%$ requirieron suplemento con Fio $2>0.4$ por más de cuatro horas y $19 \%$ ventilación mecánica, lo cual difiere con este trabajo.
La insuficiencia renal aguda se identifica cuando la concentración sérica de creatinina es $1.5 \mathrm{mg} / \mathrm{dl}$, y/o la producción de orina es menor de $0.5 \mathrm{ml} / \mathrm{k} /$ hora. $^{7} \mathrm{Sin}$ embargo, lo que ocurre después de AP puede ser no oligúrica. ${ }^{17}$ Solo dos casos desarrollaron oliguria, ambos con creatinina sérica inferior a $1 \mathrm{mg} / \mathrm{dl}$, no se encontró valor superior a $1.5 \mathrm{mg} / \mathrm{dl}$ a las 24 horas en ninguno, hallazgo que no se correlaciona con los resultados de otros autores donde hasta $70 \%$ de los afectados con AP tiene compromiso renal. ${ }^{21}$ Ancel, Garcia y colaboradores ${ }^{18}$ reportan alteración en el $42 \%$ de los pacientes, de los cuales $15 \%$ elevaron la creatinina.

La elevación de la enzima lactato deshidrogenasa es significativa en todos los casos en los que se estudió, lo cual indica compromiso muscular y hepático; concomitante con la elevación de transaminasas, ningún paciente manifestó signos clínicos ni paraclínicos de falla hepática, es importante tener en cuenta que las transaminasas permanecen elevadas varios días después del nacimiento y se deben realizar controles posteriores. ${ }^{18,23}$ Estos resultados son similares a los reportes en la literatura, Phelan y colaboradores ${ }^{10}$ encontraron un $84 \%$ comparado con $80 \%$ por Hankins y colaboradores. ${ }^{22}$

No hubo enterocolitis necrosante como también lo observaron Ancel, Garcia y colaboradores y la presencia de alteración gastrointestinal como es el residuo, se observó en $29 \%$ de los casos. ${ }^{18}$ En el presente estudio, se observa hipoglicemia en el $3.8 \%$ del total de pacientes, sin poder determinar si la severidad de la asfixia, presentada por nivel de $\mathrm{pH}$, Apgar y compromiso neurológico, están relacionados con el consumo de glucosa. 


\section{Conclusiones}

Aunque la acidosis ha sido considerada la mejor evidencia de la AP en nuestro estudio, los pacientes con mayor afectación de órganos tuvieron un $\mathrm{pH}>7.00$ incluidos cuatro de los cinco pacientes fallecidos. Algunas publicaciones han demostrado una relación entre el Apgar y la morbilidad a corto plazo después de la AP, siendo un predictor fiable de déficit neurológico permanente. En los dos pacientes con registro de alteración neurológica presentaron Apgar $<5$ en el quinto minuto.

Respecto a los cinco fallecidos, todos presentaron Apgar de 5 o menos al primer minuto, uno de ellos con puntuación 0 con $\mathrm{pH}$ de 6.96 , peso de $2.490 \mathrm{~g}$, alteración de nueve sistemas (Sarnat alterado, oliguria, ventilacion mecánica) ocurriendo la muerte al tercer día de vida. Los restantes cursaron con $\mathrm{pH}$ de 7.00, 7.16 y 7.18 ; tres de ellos con peso superior a 3.000 g y uno con $1.760 \mathrm{~g}$, el menor valor de los incluidos en el estudio. La edad gestacional promedio fue de 38 semanas. El fallecimiento se produjo en un promedio de 7.8 días. En cuatro pacientes con depresión moderada se registraron mínimo cuatro alteraciones. Son necesarios más estudios para concluir si el grupo con $\mathrm{pH}$ entre 7.00 y 7.18 tiene similar riesgo de morbimortalidad al grupo de $\mathrm{pH}<7.00$. Los resultados indican la necesidad de estudiar y vigilar los pacientes con DN moderada; se debe evaluar el efecto en todos los órganos y considerar la instauración de medidas terapéuticas contemplado la farmacocinética de los medicamentos en estos sistemas disfuncionales.

\section{Limitaciones}

En este trabajo tuvimos como limitante inicial la recolección de datos incompleta, lo que implicó una pérdida de pacientes que cumplían los criterios de inclusión. La realización de este estudio en el futuro próximo a nivel multicéntrico en el Hospital de San José y el Hospital Infantil Universitario de San José, permitirá obtener mayor número de pacientes y de esta manera evaluar otras variables y comparar los dos grupos.

\section{Referencias}

1. Leuthner SR, Das UG. Low Apgar scores and the definition of birth asphyxia Pediatr Clin North Am. 2004; 51:737-45.

2. McGuire W. Perinatal asphyxia. Clin Evid (On!ine). 2007 Nov 7:2007. pii: 0320.

3. Blickstein I, Green T. Umbilical cord blood gases. Clin Perinatol. 2007;34:451-9.

4. Gupta BD, Sharma P, Bagla J. Parakh M, Soni JP. Renal failure in asphyxiated neonates. Indian Pediatr. 2005; 42:928-34.

5. Leone TA, Finer NN. Shock: a common consequence of neonatal asphyxia. J Pediatr. 2011;158:c9-12.

6. Young CM, Kingma SD, Neu J. Ischemia-reperfusion and neonatal intestinal injury. J Pediatr. $2011 ; 158:$ e25-8.

7. Lapointe A, Barrington KJ. Pulmonary hypertension and the asphyxiated new born. J Pediatr. 2011;158:el9-24.

8. Bauman ME, Cheung PY, Massicotte MP. Hemostasis and platelet dysfunction in asphyxiated neonates. J Pediatr. 2011;158:e35-9.

9. Al-Macki N, Miller SP, Hall N, Shevell M. The spectrum of abnormal neurologic outcomes subsequent to term intrapartum asphyxia. Pediatr Neuro.1 2009;41:399405 .

10. Phelan JP, Martin GI, Korst LM. Birth asphyxia and cerebral palsy. Clin Perinatol. 2005;32:61-76, vi.

11. Pin TW, Eldridge B, Galea MP. A review of developmental outcomes of term infants with post-asphyxia neonatal encephalopathy. Eur J Paediatr Neurol. 2009;13:224-34

12. Tarcan A, Tiker F, Güvenir H, Gürakan B. Hepatic involvement in perinatal asphyxia. J Matern Fetal Neonatal Med. 2007;20:407-10.

13. Fischler B, Pettersson M, Hjern A, Nemeth A. Association between low Apgar score and neonatal cholestasis. Acta Paediatr. 2004;93:368-71.

14. Kluckow M. Functional echocardiography in assessment of the cardiovascular system in asphyxiated neonates. J Pediatr. 2011;158:el3-8.

15. Costa S, Zecca E, De Rosa G, et al. Is serum troponin T a useful marker of myocardial damage in newborn infants with perinatal asphyxia? Acta Paediatr. 2007;96:181-4

16. Rajakumar PS, Vishnu Bhat B, Sridhar MG, et al. Electrocardiographic and echocardiographic changes in perinatal asphyxia. Indian J Pediatr. 2009;76:261-4.

17. Durkan AM, Alexander RT. Acute kidney injury post neonatal asphyxia. J Pediatr. 2011;158:e29-33.

18. Martín-Ancel A, García-Alix A, Gayá F, Cabañas F, Burgueros M, Quero J. Multiple organ involvement in perinatal asphyxia. J Pediatr. 1995;127:786-93.

19. Basu P, Som S, Choudhuri N, Das H. Contribution of the blood glucose level in perinatal asphyxia. Eur J Pediatr. 2009;168:833-8.

20. Aggarwal R, Upadhyay M, Deorari AK, Paul VK. Hypocalcemia in the newborn Indian J Pediatr. 2001;68:973-5

21. Shah P, Riphagen S, Beyene J, Perlman M. Multiorgan dysfunction in infants with post-asphyxial hypoxic-ischaemic encephalopathy. Arch Dis Child Feta Neonatal Ed. 2004;89:F152-5.

22. Hankins GD, Koen S, Gei AF, Lopez SM, Van Hook JW, Anderson GD. Neonatal organ system injury in acute birth asphyxia sufficient to result in neonata encephalopathy. Obstet Gynecol. 2002;99:688-91.

23. Karlsson M, Blennow M, Nemeth A, Winbladh B. Dynamics of hepatic enzyme activity following birth asphyxia. Acta Paediatr. 2006;95:1405-11. 